\title{
Photoacoustic Doppler flowmetry
}

\section{Hui Fang, Lihong V. Wang}

Hui Fang, Lihong V. Wang, "Photoacoustic Doppler flowmetry," Proc. SPIE 6856, Photons Plus Ultrasound: Imaging and Sensing 2008: The Ninth Conference on Biomedical Thermoacoustics, Optoacoustics, and Acoustooptics, 68561M (28 February 2008); doi: 10.1117/12.761947

SPIE. Event: SPIE BiOS, 2008, San Jose, California, United States 


\title{
Photoacoustic Doppler flowmetry
}

\author{
Hui Fang and Lihong V. Wang* \\ Optical Imaging Laboratory, Department of Biomedical Engineering, \\ Washington University in St. Louis, St. Louis, Missouri 63130, USA
}

\begin{abstract}
We report flow measurements based on the photoacoustic Doppler effect. We have performed flow experiments with a suspension of micrometer carbon particles and have detected the photoacoustic Doppler shift at various average flow speeds. We have also observed the directional dependence of the photoacoustic Doppler shift. Our experiment is based on the continuous wave $(\mathrm{cw})$ photoacoustic generation. It is the goal of noninvasively monitoring hemodynamics in functional photoacoustic imaging that motivates our study.
\end{abstract}

Keywords: Flow measurement, Doppler effect, photoacoustic imaging, photoacoustic Doppler effect, hemodynamics

\section{INTRODUCTION}

In recent years, photoacoustic imaging has emerged as a unique imaging modality to study microvascular structures. ${ }^{1}$ With the benefits of both one to two orders of magnitude higher light absorption of blood than the surrounding tissue and two to three orders of magnitude weaker scattering of ultrasound than light in biological tissues, ${ }^{2}$ it can examine blood vessels at the depth of a few millimeters with the spatial resolution of tens of micrometers. Moreover, with the developments of both the linear array transducer video imaging system ${ }^{3}$ and the multiple wavelength spectroscopic imaging system, ${ }^{4}$ it can noninvasively monitor the changes of blood volume and hemoglobin oxygenation saturation.

Despite these dramatic developments, the question in photoacoustic imaging to obtain the information about microvascular blood flow speed, which is necessary for the complete description of microvascular hemodynamics, has yet waited to be addressed. Considering the incomparable advantages of photoacoustic imaging, it will be a great interest to investigate if the blood flow can be detected in such a unified approach. Although the efforts have been taken to measure flow with gold nanoparticles as exogenous photoacoustic contrast agent, ${ }^{5}$ we are more interested in developing a noninvasive method to measure blood flow by using red blood cells as endogenous photoacoustic contrast particles. This goal motivated us to study the photoacoustic Doppler effect from moving particles.

\section{PHOTOACOUSTIC DOPPLER EFFECT}

Conventional laser Doppler and acoustic Doppler flowmetry are both scattering-based Doppler methods in the sense that they both require the presence of small scattering particles (scatter either optical or acoustic waves) to provide Doppler shifted backscattering signals. The flowmetry that we are developing is based on the photoacoustic Doppler effect from moving particles and is totally different from the conventional Doppler flowmetry. It uses light absorbing particles to provide Doppler shifted photoacoustic signals. As illustrated in Fig. 1, a moving light absorbing particle will transfer the laser energy to the form of an acoustic wave. ${ }^{7} 8$ The acoustic wave should have a Doppler shift added to the original laser intensity modulation frequency because of the particle motion.

It is our surprise that the discussion about the photoacoustic Doppler effect from moving particles has not been found in literature before our recent study.,10 To our best acknowledge, only the photoacoustic Doppler effect from laser scanning has been previously studied in a few researches. ${ }^{11-15}$ The basic idea of those researches is that when an

*1hwang@biomed.wustl.edu; phone: 314 935-6152; fax: 314 935-7448

Photons Plus Ultrasound: Imaging and Sensing 2008: The Ninth Conference on Biomedical Thermoacoustics, Optoacoustics, and Acousto-optics, edited by Alexander A. Oraevsky, Lihong V. Wang, Proc. of SPIE Vol. 6856, 68561M, (2008) · 1605-7422/08/\$18 - doi: 10.1117/12.761947 
intensity-modulated laser is scanned across a water surface, a moving thermoacoustic array is created inside the water due to thermoacoustic effect (or equivalently photoacoustic effect) and an acoustic wave is emitted by the array. The acoustic wave owns a frequency Doppler-shifted from the modulation frequency. There, the Doppler shift depends on the speed of laser scanning and no flow of media is involved.

\section{PRINCIPLE OF PHOTOACOUSTIC DOPPLER FLOWMETRY}

Photoacoustic Doppler flowmetry needs small light absorbing tracer particles to detect a fluid flow. The particles should have small size, low volume fraction, and appropriate mass density so that they will not change the flow condition and can flow along with the fluid without any sliding. To model a real flow which usually has a complex flow profile, the photoacoustic Doppler signals from many particles needs to be considered. For simplicity, in this paper, we only consider the case of one single moving particle, as illustrated in Fig.1.

\section{Ultrasonic Transducer}

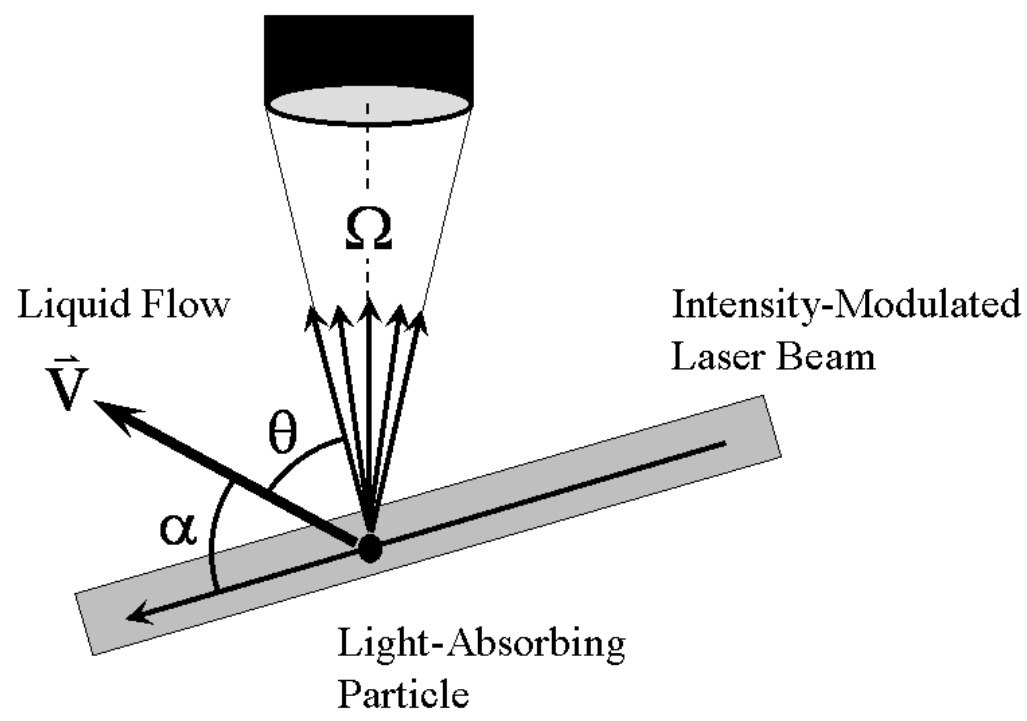

Fig. 1 Schematic of the photoacoustic Doppler effect from a moving particle.

As it is shown in Fig.1, when a light-absorbing particle in a flowing liquid passes through the illumination area of an intensity-modulated laser beam, an acoustic wave will be generated due to the continue wave photoacoustic effect and can be detected by an ultrasonic transducer. The process of the acoustic wave generation is as following: ${ }^{8}$ first, a periodic heat will be produced after the particle absorbs laser energy; subsequently, the heat will dissipate to the surrounding liquid, cause an oscillatory motion of a thin layer of liquid at the particle-liquid boundary, and finally produce an acoustic wave. Because the particle is in motion, the frequency of resulted acoustic wave should have a Doppler shift. Before deriving the equation of the Doppler shift, let us first write down the amplitude of the acoustic wave. According to the theory of the photoacoustic effect from a stationary solid particle, ${ }^{8}$ the amplitude can be simplified as

$$
\left|p_{A}\right|=\frac{\bar{\alpha} \cdot I_{O} \cdot A_{P} \cdot a^{3} \cdot 2 \pi f_{M}}{r}, \text { for } \frac{2 \pi f_{M} \cdot a}{c_{L}}<<1
$$


where $\mathrm{r}$ is the distance from the particle, $\bar{\alpha}$ the absorption coefficient of the particle, $I_{O}$ the intensity of the illumination laser, $A_{P}$ a constant related to the physical properties of the particle which is equal to $\frac{K \beta}{C_{P} \rho_{s}\left(\rho_{s} / \rho_{f}\right)\left(3 c_{L}^{2}-4 c_{T}^{2}\right)}$ (where $K$ is the bulk modulus, $\beta$ the thermal expansion coefficient, $C_{P}$ the heat capacity, $\rho_{s}$ the mass density and $\rho_{s} / \rho_{f}$ the density ratio relative to the surrounding liquid, $c_{L}$ the longitudinal sound speed, and $c_{T}$ the transverse sound speed.), $\quad a$ the particle radius, and $f_{M}$ the modulation frequency of the laser.

To calculate the Doppler shift, we need to consider the detail geometry including the flow direction, the laser illumination, and the ultrasonic detection. As shown in Fig.1, the flow is along velocity $\vec{V}$, the laser beam has an angle $\alpha$ with respect to the flow, and the acoustic wave is collected inside the solid angle $\Omega$. At the angle $\theta$ which represents one of the general direction with respect to the flow, the Doppler shift of the acoustic wave which is called by us as the photoacoustic Doppler shift (PAD shift), can be expressed as:

$$
\begin{aligned}
f_{\mathrm{PAD}} & =-f_{M} \frac{v \cdot \cos \alpha}{c_{O}}+f_{M} \frac{v \cdot \cos \theta}{c_{A}} \equiv-\frac{v \cdot \cos \alpha}{\lambda_{O}}+\frac{v \cdot \cos \theta}{\lambda_{A}} \\
& \approx f_{M} \frac{v \cdot \cos \theta}{c_{A}}
\end{aligned}
$$

where $c_{O}$ and $c_{A}$ are the speeds of light and sound in the liquid, respectively, $\lambda_{O}$ and $\lambda_{A}$ are the wavelengths of the laser photon density wave and the acoustic wave, respectively. Because $\lambda_{O}$ is much longer than $\lambda_{A}$, for the usual case when $\theta$ is not very close to $90^{\circ}$, only the term finally left is important. Because the detection has an angular distribution inside the solid angle $\Omega$, the actual PAD shifts will have a distribution as well. The mean PAD shift should be calculated by the integral including all possible detection angles.

\section{PHOTOACOUSTC DOPPLER FLOW MEASURING EXPERIMENTS}

Our experiment system is developed from the previously home-built continuous-wave photoacoustic microscope and has been detailed in the recently publications. ${ }^{9,10}$ Here, we will briefly describe the system using the function block diagram plotted in Fig.2. Started from the function generator, it provides an amplitude-modulated sine wave to modulate the intensity of the continuous wave laser. The laser beam illuminates the flowing fluid thus the light absorbing tracer particles in it. The acoustic wave is collected by the ultrasonic transducer and the photoacoustic signal is input to the quadrature demodulator. The function generator also provides the reference input into the demodulator, which is a sine wave with the same frequency as the laser modulation. We currently use a lock-in detector as the quadrature demodulator. From the demodulator, the acoustic signal is transformed to a pair of demodulated outputs. A spectral analysis yields the PAD power spectra and thus the PAD shifts. By the theoretical modeling based on Eq. (2), flow information such as the average flow speed, flow profile, and flow direction can be extracted.

The fluid flow was generated by a syringe pump (BSP-99M, Braintree Scientific) and a Tygon ${ }^{\circledR}$ tube (inside diameter: $0.51 \mathrm{~mm}$; outside diameter: $1.53 \mathrm{~mm}$; S-54-HL, Saint-Gobain Performance Plastics). Through the syringe pump, the flow speed could be set manually. The flow samples were made from the carbon particles with diameters distributed from 2 to 12 micrometers (carbon glassy spherical powder, 484164, Sigma-Aldrich). The solution for suspending the particles was made by dissolving an appropriate amount of solid sodium polytungstate (Sometu) into distilled water so that its mass density became about $1.46 \mathrm{~g} / \mathrm{cm}^{3}$, which matched that of the particles. Also, $1 \%$ volume of Tween-20 (Sigma-Aldrich) was added into the solution to reduce particle aggregation.

We have performed the experiments with the suspension samples which have the particle volume fraction ranged from $10 \%$ to $40 \%$. It was found that the mean PAD shifts do not depend on the volume fractions. We also compared the experiment results measured in scattering medium and in clear medium where the clear medium is distilled water and the scattering medium is the diluted intralipid solution with concentration $0.04 \%$ (scattering coefficient $\mu_{s}$ about 1 
$\mathrm{cm}^{-1}$ ). In such a scattering medium when measured at the depth about $3 \mathrm{~cm}$, the magnitude of the PAD signals decrease about 10 times due to the multiple light scattering, compared to the signals in the clear medium. However, we found that the mean PAD shifts keep the same.

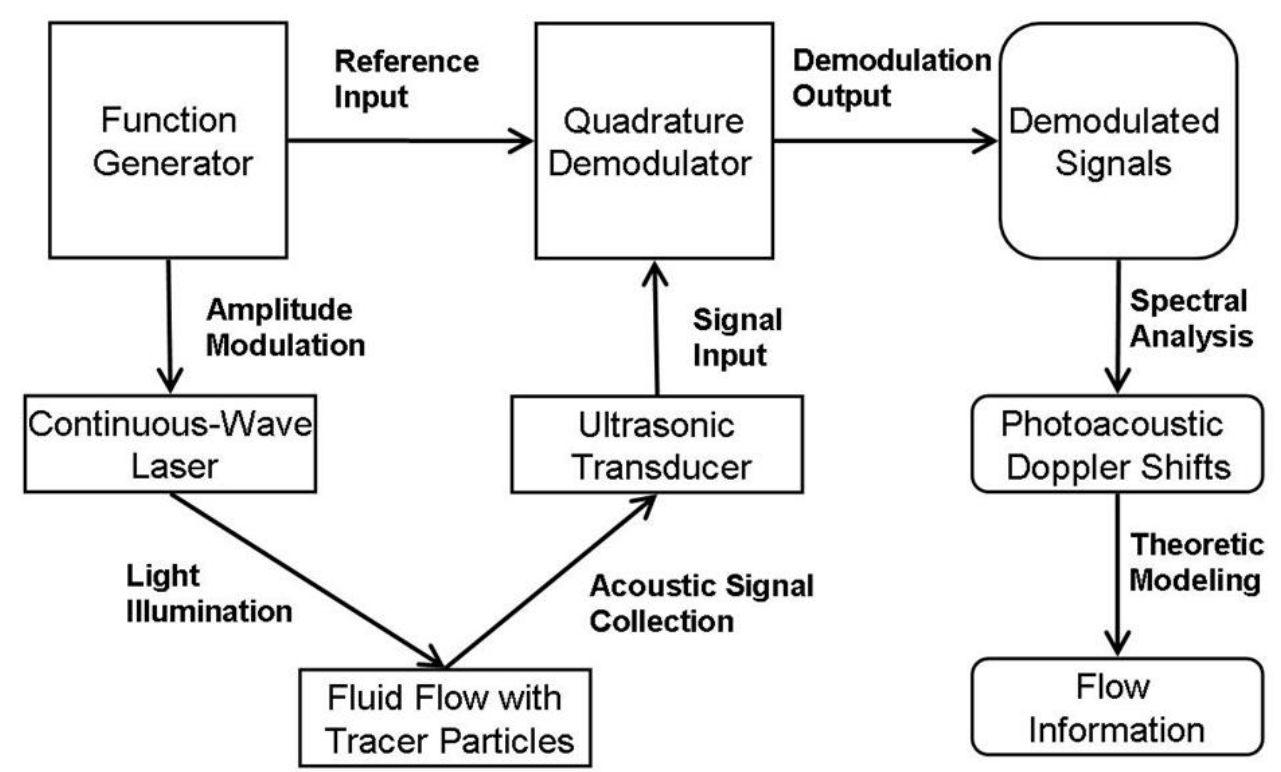

Fig.2 Function block diagram of the photoacoustic Doppler flow measuring system.

\section{EXPERIMENT RESULTS}

\subsection{Photoacoustic Doppler shift versus flow speed}

We studied PAD shifts at different flow speeds. As shown in Fig. 3, the mean PAD shifts in the scattering media are plotted as the circular date points, the mean PAD shifts in the clear medium are plotted as the square data points, and the theoretical calculation is plotted as the line. The calculation uses the equation of

$$
\bar{f}_{\mathrm{PAD}}=\frac{\int_{0^{0}}^{60^{0}} f_{\mathrm{PAD}} \times 2 \pi R \sin \theta d \theta}{\int_{0^{0}}^{60^{0}} 2 \pi R \sin \theta d \theta}=f_{M} \frac{v}{c_{A}} \frac{\left(1+\cos 60^{\circ}\right)}{2}
$$

with $f_{M}=2.455 \mathrm{MHz}$ and $c_{A}=1500 \mathrm{~m} / \mathrm{s}$. The calculation corresponds to the detection geometry where the transducer has a numerical aperture $N A=\sin \left(60^{\circ}\right)$ and the flow is along the ultrasonic transducer's axis.

As can been seen, the mean PAD shifts both in the scattering and clear medium can be accurately described by the theoretical calculation Eq. (3). This demonstrated that the PAD shifts are not affected by the multiple light scattering. Also, the minimum detectable flow speed is not affected as well. On the other hand, the maximum detectable flow speed thus the detectable range has decreased 10 times from about $10 \mathrm{~mm} / \mathrm{s}$ to $1 \mathrm{~mm} / \mathrm{s}$. This is because the multiple light scattering reduces the laser intensity thus decreases the photoacoustic signal. The maximum measurable flow speed was limited by the signal-to-noise ratio. When the flow speed increased, the PAD power spectrum broadened 
linearly. At the same time, the PAD spectral amplitude decreased and eventually approached the noise level. In principle, based on Eq. (1), the range can be expanded by the boost of laser intensity $I_{O}$, the increase of the modulation frequency $f_{M}$, the enhance of the particle's light absorption $\bar{\alpha}$, and the increase of particle radius $a$ or the particle's physical property $A_{P}$. The range can also be expanded by optimizing the ultrasonic detection such as the detection distance $r$, the sensitivity of the transducer.

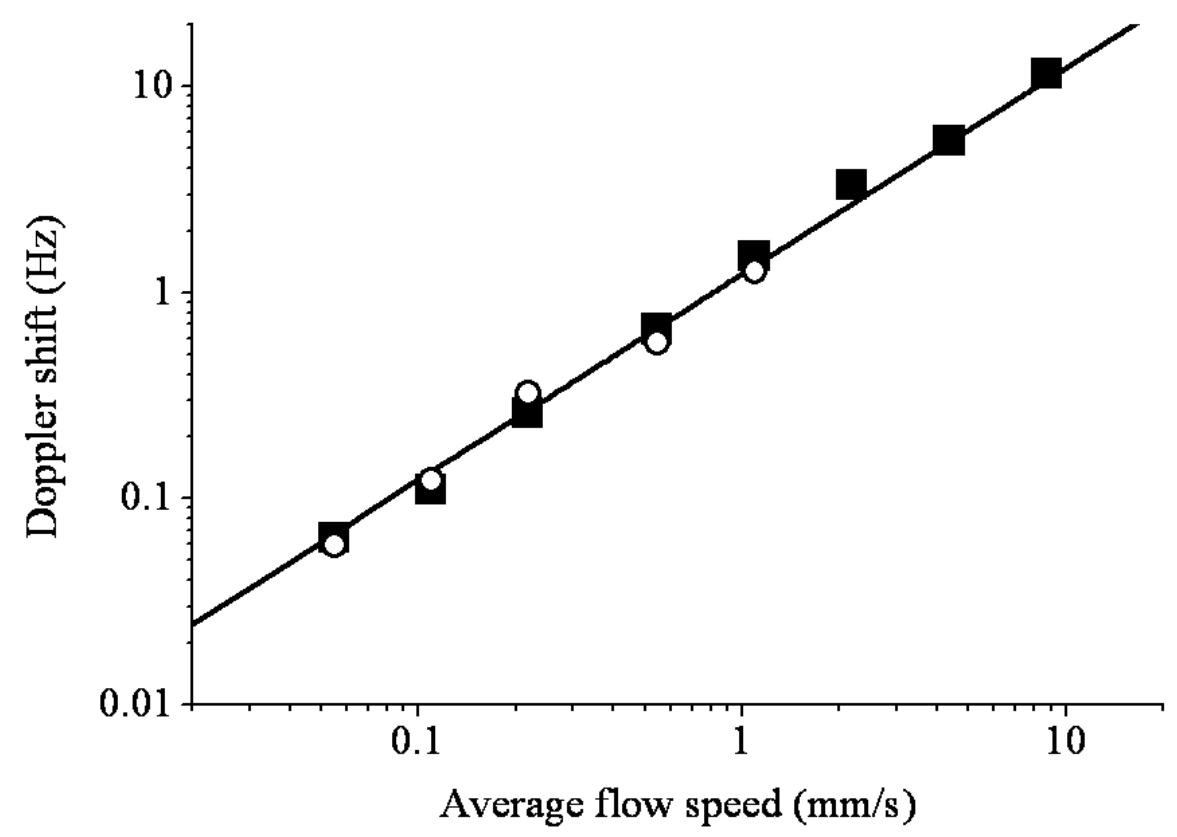

Fig. 3 Mean photoacoustic Doppler shifts measured at various average flow speeds. The square data points plot the measurement results in the clear medium; the circular data points plot the measurement results in the scattering medium; the line plots the theoretical calculation.

\subsection{Photoacoustic Doppler shift versus flow direction}

Because the PAD shift, as expressed in Eq. (2), does not depend on the laser illumination geometry, the flow directional information can be extracted even in the scattering media, potentially by detecting along multiple angles. We have demonstrated the observation of PAD shifts with opposite signs by measuring flow with reversed flow directions. The positive shift is measured from the flow towards the transducer and the negative shift from the flow away from the transducer. Since the lock-in detector uses phase sensitive detection to produce a pair of quadrature-demodulated outputs $\mathrm{X}$ and $\mathrm{Y}$ (where $\mathrm{X}$ is the low-pass filtered product of the signal and the reference, and $\mathrm{Y}$ is the low-pass filtered product of the signal and the $+90^{\circ}$ phase shifted reference, the positive and negative shift can be separated by the phase information embedded in the $\mathrm{X}$ and $\mathrm{Y}$. The ac components of $\mathrm{X}$ and $\mathrm{Y}$ can be expressed as:

$$
\begin{aligned}
& X_{-\mathrm{ac}}=\bar{A}_{D} \cos \left(2 \pi \bar{f}_{\mathrm{PAD}} t+\psi_{D}\right) \\
& Y_{-\mathrm{ac}}=\bar{A}_{D} \cos \left(2 \pi \bar{f}_{\mathrm{PAD}} t+\psi_{D}-\pi / 2\right)
\end{aligned}
$$

where $\bar{A}_{D}$ and $\psi_{D}$ denote the amplitude and initial phase, respectively, of the PAD-shifted acoustic signal. For positive $\bar{f}_{\mathrm{PAD}}$, Y should lag X by $\pi / 2$ while for negative $\bar{f}_{\mathrm{PAD}}, \mathrm{Y}$ should lead $\mathrm{X}$ by $\pi / 2$. Figure 4 shows the 
directional discrimination results. The signals are measured from the flow in the scattering medium with the average flow speed of $0.27 \mathrm{~mm} / \mathrm{s}$, which corresponds to the mean PAD shift of $0.32 \mathrm{~Hz}$.
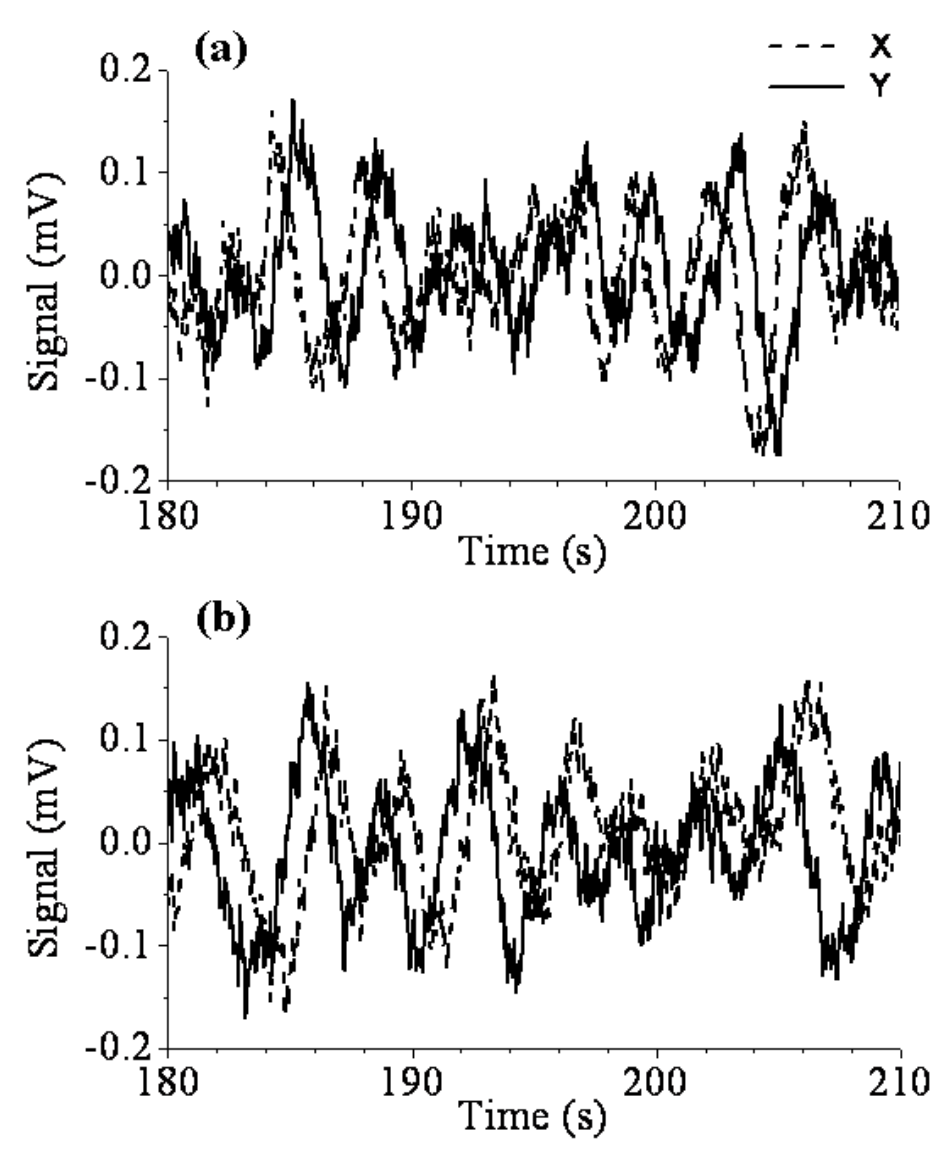

Fig. 4 Directional discrimination of photoacoustic Doppler signals measured in the scattering medium. Average flow speed: $0.27 \mathrm{~mm} / \mathrm{s}$. (a) ac components of $\mathrm{X}$ and $\mathrm{Y}$ signals for a flow toward the transducer. (b) ac components of $\mathrm{X}$ and $\mathrm{Y}$ signals for a flow away from the transducer.

\section{DISCUSSION}

In our current study, we have used the suspension of micrometer carbon particles with very high particle volume fractions. This is because we are interested in measuring the flow of blood where red blood cells have similar volume fractions as well as similar sizes. In general, in order to study a flow by applying exogenous light absorbing tracer particles, volume fraction of the particles should be as low as possible and the particle size can be as small as possible, depending on the sensitivity of photoacoustic signal detection. Otherwise, the flow condition may be changed because of introducing the particles.

Photoacoustic Doppler flowmetry has following several apparent advantages compared to the conventional Doppler flowmetry because of its two major properties. One is that it uses light absorbing particles instead of scattering particles as the flow tracer. Consequently, it could have much low background noise and much higher detection contrast. Ideally, if only the tracer particles absorb light, the photoacoustic Doppler signal should only come from these particles. It 
avoids the problem that the reflection from the flow wall with slow motion always masks the Doppler signal from low speed scattering particles near the wall. Acoustic flowmetry is limited by this problem and usually has difficulty to measure a low speed flow. The other is that it employs photon density wave instead of optical wave to provide the frequency. As results, it will not suffer from the multiple light scattering inside the flow surrounding medium. It can provide correct Doppler shifts and directional discrimination even for the flow at large depth inside a scattering medium. Laser Doppler flowmetry has a limited measuring depth and loses the flow directional information, both due to the multiple light scattering.

\section{FUTURE APPLICATONS}

Because of the advantages just mentioned, photoacoustic Doppler flowmetry could open a new window for the flow measurement, especially for studying the flow with a very low speed and in a very small channel. It can also provide incomparable capability to detect low speed flows with high contrast in highly scattering media at large depth. In our lab, we are interested to develop this technique to measure blood flow in microcirculation which is still a challenge for other Doppler methods because of microcirculation's small vessel size $(\sim 5-100 \mu \mathrm{m})$, low blood flow speed $(<50 \mathrm{~mm} / \mathrm{s})$, and microvascular structure depth (up to $\sim 5 \mathrm{~mm}$ ). ${ }^{16}$ Studying this type of blood flow is very important for understanding several major diseases such as diabetes and cancer. ${ }^{17}$ We anticipate that Photoacoustic Doppler flowmetry could become a very good solution for studying microcirculation.

\section{ACKNOWLEDGEMENT}

We would like to acknowledge the support provided by National Institutes of Health under Grant Nos. R01 EB000712 and R01 NS046214. We also would like to acknowledge the contribution from Dr. Konstantin Maslov and the help from Dr. Geng Ku.

\section{REFERENCES}

[1] C. G. A. Hoelen, F. F. M. de Mul, R. Pongers, and A. Dekker, "Three-dimensional photoacoustic imaging of blood vessels in tissue," Opt. Lett. 23, 648-650 (1998).

[2] K. Maslov, G. Stoica, and Lihong V. Wang, "In vivo dark-field reflection-mode photoacoustic microscopy," Opt. Lett. 30, 625-627 (2005).

[3] D. W. Yang, D. Xing, S. H. Yang, L. Z. Xiang, "Fast full-view photoacoustic imaging by combined scanning with a linear transducer array," Opt. Express 15, 15566-15575 (2007).

[4] H. F. Zhang, K. Maslov, G. Stoica and L. V. Wang, "Functional photoacoustic microscopy for high-resolution and noninvasive in vivo imaging," Nat. Biotechnol. 24, 848-851 (2006).

[5] W. W. Chen, C. K. Liao, H. C. Tseng, Y. P. Lin, C. C. Chen, and P. C. Li, "Photoacoustic flow measurements with gold nanoparticles," IEEE Trans. Ultrason., Ferroelect., Freq. Contr. 53, 1955-1959 (2006).

[6] R. J. Goldstein, Ed., [Fluid Mechanics Measurements], Taylor \& Francis, Bristol (1996).

[7] G. J. Diebold, A. C. Beveridge, and T. J. Hamilton, "The photoacoustic effect generated by an incompressible sphere," J. Acoust. Soc. Am. 112, 1780-1786 (2002).

[8] M. I. Khan and G. J. Diebold, "The phtoacoustic effect generated by an isotropic solid sphere," Ultrasonics 33, 265-269 (1995).

[9] H. Fang, K. Maslov, and L. V. Wang, "Photoacoustic Doppler effect from flowing small light-absorbing particles," Phys. Rev. Lett. 99, 184501-(1-4) (2007).

[10] H. Fang, K. Maslov, and L. V. Wang, "Photoacoustic Doppler flow measurement in optically scattering media," Appl. Phys. Lett. 91, 264103-(1-3) (2007).

[11] A. I. Bozhkov, F. V. Bunkin, and Al. A. Kolomenskii, "Doppler thermooptical source of ultrasound," Sov. Phys. Acoust. 25, 443-445 (1979). 
[12] Al. A. Kolomenskii, "Doppler signal and Mach waves in the far field of a two-dimensional thermoacoustic radiator," Sov. Phys. Acoust. 33, 526-528 (1987).

[13] Y. H. Berthelot and L. J. Busch-Vishniac, "Thermoacoustic radiation of sound by a moving laser source," J. Acoust. Soc. Am. 82, 317-327 (1987).

[14] N. P. Chotiros, "The moving thermoacoustic array: a theoretical study," J. Acoust. Soc. Am. 83, 2145-2158 (1988).

[15] Y. H. Berthelot, "Experimental investigation of the sound field generated by a moving ruby-laser thermoacoustic array," J. Acoust. Soc. Am. 83, 1399-1405 (1988).

[16] D. E. Goertz, J. L. Yu, R. S. Kerbel, P. N. Burns, and F. S. Foster, "High-frequency 3-d color-flow imaging of the microcirculation," Ultrasound in Med. and Biol. 29, 39-51 (2003).

[17] B. Fagrell and M. Intaglietta, "Microcirculation: its significance in clinical and molecular medicine," J. Intern Med. 241, 349-362 (1997). 